EPiC Series in Engineering
Volume 3, 2018, Pages 1081-1087
HIC 2018. 13th International
Conference on Hydroinformatics

\title{
Designing a framework for the estuarine monitoring system
}

\author{
Nam-Hoon, Kim ${ }^{1}$ and Jin Hwan Hwang ${ }^{1 *}$ \\ ${ }^{1}$ Seoul National University, Seoul, South Korea \\ nhkim0426@snu.ac.kr, jinhwang@snu.ac.kr
}

\begin{abstract}
Where the physical and environmental characteristics of the coast and estuary change dramatically and complicatedly due to the multiple controlling sources as like as the tide, wave, river discharge, in order to collect the environmental data effectively and reliably, the locations of monitoring should be optimally determined with the standardized framework. The present study proposes a protocol to find the best monitoring location in a local bay based on a spatial and temporal optimizing method. W ith the simulation data from the accurately validated numerical model, the monitoring locations are designed with the constrained optimization method, which finds the optimal solution by constraining the objective function into the design space. The objective and constrained functions are determined from the objective analysis, which is combined with constrained optimization. Finally, those two functions are used to find the optimal solutions of the locations.
\end{abstract}

\section{Introduction}

The physical and environmental characteristics of a bay should be monitored considering the environmental variability of them $[12,16]$. In particular, since an estuarine system has a lot of control factors, which affect its environmental and ecological conditions, the environmental monitoring in the estuary need to be optimally determined considering the variations of all factors as like as tide, wave, geomorphology, discharge of freshwater etc [11]. Optimal designing can help reducing the costs and the efforts in operation and management of monitoring systems and increasing the reliability of the measurement data. [15] carried out a study on how many drifters are required to describe meso-scale features in the Azores-Madeira region and [3] optimized the idealized cases to configure a system of the acoustic tomography array. But the many locations of monitoring have been decided by the researcher's common sense rather than the scientific approach. Therefore, there have been few studies designing the optimal monitoring systems, in particular, to be applicable to deploy the long-term

*Corresponding author: jinhwang@snu.ac.kr 
monitoring systemin rather small and complicate systemas like as the Korean coastalareas. Moreover, the standard protocol has not been proposed for determining the numbers and locations of sensors and durations and frequencies of sampling along with effectively representing the characteristics of the target area. Therefore, this study proposes a framework of the optimization for determining the locations of monitoring and examines the changes of temporal and spatial distributions of the physical and environmental characteristics as an application to the real site with the results from the numerical simulations.

\section{Material and methods}

The Geum River Estuary (hereafter GRE) is located in the east part of the Yellow Sea and the west coast of Korea [Figure 1(a)] [8]. Artificial gates at the sea-dike discharge from the Geum River to the coastal sea irregularly, depending on the differences of the water level between inside and outside of the coastal reservoir. Freshwater discharge controls a lot the physical and environmental characteris tics such as stratification, bottom and interfacial shears, phosphorus, nitrogen, dis solved oxygen, $\mathrm{pH}$, etc. [9]. The GRE, in particular, has a strong variability of the salinity due to the freshwater discharge and the interpretation of the relationship between the other variables is complicated. Therefore, this area should be operated by an appropriate monitoring system. To optimize the monitoring system, the present work uses the simulation results of [5]. The simulation reproduced the physical and environmental variables of the GRE with the three-dimensional hydrodynamic model of Delft-3D [Figure 1(b)], which was carefully downscaled from the larger scale model [10, 17].

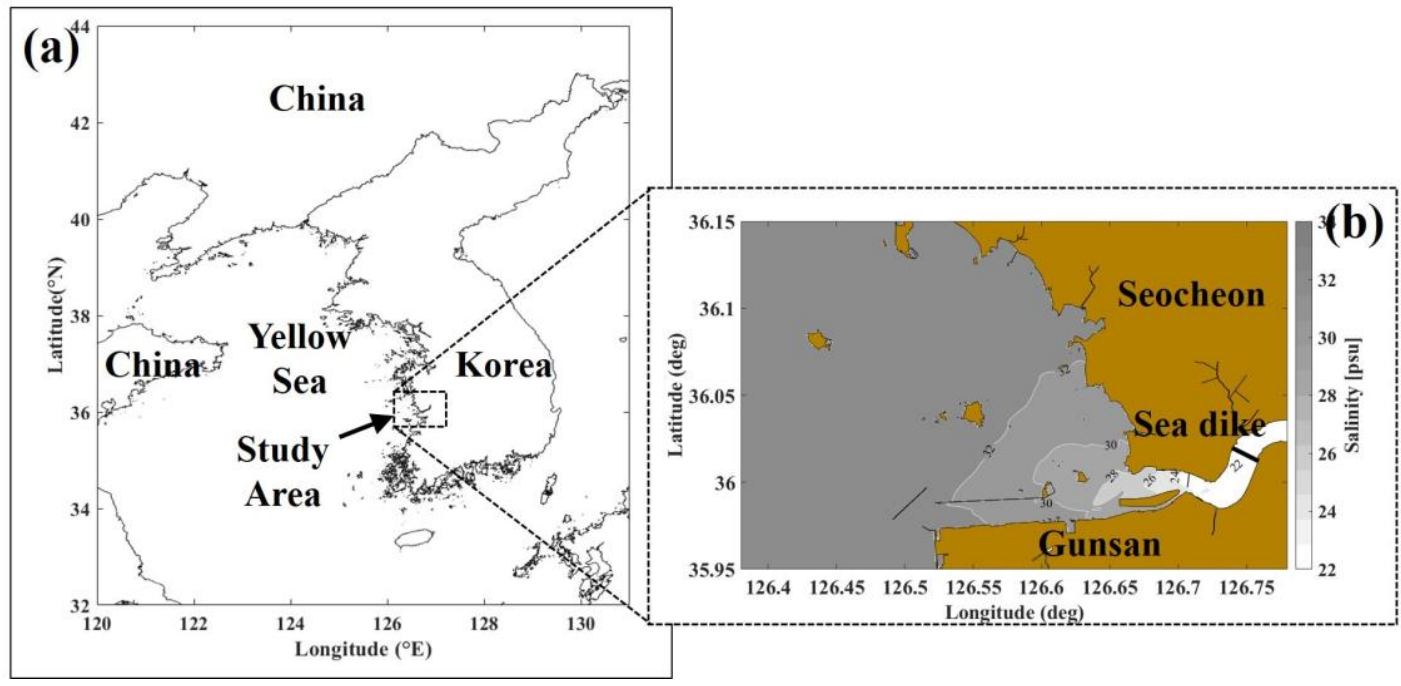

Figure 1: (a) Location of study area and (b) an example of the salinity distribution simulated by Delft-3D [5]

\subsection{Application of optimization technique}

Graphical optimization is a method obtaining the solution with the objective function [4, 18]. In many graphical optimizations, one of the methods is the constraint optimization, which finds the 
solutions by constructing an objective function into the constraint regions. In the several ways to find the solutions, the transformation method is the simplest and most popular to handle the constraints [13]. In this method, the local constraint problem is transformed into a sequence of an unconstrained problem by adding the penalty terms for each violation of constraints. The penalty terms are referred to the penalty function and this study, we applied the Exterior Penalty Function (EPF) method, which is easy to appreciate and implement [18]. After transforming a function to the unconstraint one, the function becomes:

$$
\begin{array}{ll}
\text { Minimize } & F\left(x, y, r_{h}, r_{g}\right)=f(x, y)+P\left(x, y, r_{h}, r_{g}\right) \\
\text { Subject to } & x^{\text {lower }} \leq x \leq x^{\text {upper }} ; \\
& y^{\text {lower }} \leq y \leq y^{\text {upper }} ;
\end{array}
$$

where $x$ and $y$ are the design variables and $r_{h}$ and $r_{g}$ are penalty constants (also called penalty multipliers $). f(x, y)$ is the objective function and $P\left(x, y, r_{h}, r_{g}\right)$ is the penalty function, $F\left(x, y, r_{h}, r_{g}\right)$ is referred to the augmented function. Side constraints (lower and upper bound of design variables) express the acceptable region for the design variables. All design functions explicitly or implicitly depend on the design variable. The penalty function is formulated as

$$
P\left(x, y, r_{g}, r_{g}\right)=r_{h}\left[\sum_{k=1}^{l} h_{k}(x, y)\right]+r_{g}\left[\sum_{j=1}^{m}\left(\max \left\{0, g_{j}(x, y)\right\}\right)^{2}\right]
$$

where $g(x, y)$ is the constrained function which is also expressed as the inequality constrained function. The subscript $j$ represents the $j^{t h}$ region or function of inequality constraints. The area of the design variables enclosed by the constraints is called the feasible domain. The first term of the right hand side of eq. (2) is ignored since this term represents the equality-constrained functions, which are rarely employed in the practical problems [18]. If the inequality constraint does not reach an optimal solution, new $P$ is reconstructed by increasing the penalty multipliers adding to $f$, the objective function to have new $F$, the augmented. As the solutions approach the true optimal solution, the multipliers $r_{h}$ and $r_{g}$ need to become infinite. Therefore, the conditions for converging to the optimal solution using the EPF are (1) $\Delta F^{T} \Delta F \leq \varepsilon$ (function not changing), (2) $\Delta X^{T} \Delta X \leq \varepsilon$ (design not changing), and (3) maximum iterations reached.

\subsection{Objective Analysis}

The most important part of the optimization is how to set the appropriate objective and constrained functions. The gradients or slopes of the contours for target parameters are determinant to find the optimal solutions in the graphical optimization. Therefore, for constructing the appropriate contours, the discretely sampled data should be transformed to the continuous functions with interpolation techniques such as the objective analys is. Once the continuous function is constructed, then we can find the maxima and minima. To interpolate and construct the distribution function, the objective analys is scheme of [2] is used here (hereafter BOA). This scheme can interpolate the whole region of interest by repeatedly applying a distance dependent weighting function based on the irregularly distributed reference values [14]. In BOA, the variables $D\left(x_{m}, y_{m}\right)$ are the reference values at $m$ locations and are constructed by three steps and new $D$ s are regained at each step. The first, second and third $D s$ are described as: 


$$
\begin{aligned}
& D_{g}^{1}=\frac{\sum_{m=1}^{N} w_{m} D\left(x_{m}, y_{m}\right)}{\sum_{m=1}^{N} w_{m}} ; \\
& D_{g}^{2,3}=D_{g}^{1,2}+\frac{\sum_{m=1}^{N} w_{m}^{\prime}\left[D\left(x_{m}, y_{m}\right)-D^{1,2}\left(x_{m}, y_{m}\right)\right]}{\sum_{m=1}^{N} w_{m}^{\prime}} ;
\end{aligned}
$$

where $m^{\text {th }}$ weights applied to the reference value at $g$ for each step are given by:

$$
\begin{aligned}
& w_{m}=\exp \left[-\left(\frac{d_{m x}^{2}}{c_{x}^{2}}+\frac{d_{m y}^{2}}{c_{y}^{2}}\right)\right] ; \\
& w_{m}^{\prime}=\exp \left[-\left(\frac{d_{m x}^{2}}{\gamma c_{x}^{2}}+\frac{d_{m y}^{2}}{\gamma c_{y}^{2}}\right)\right] .
\end{aligned}
$$

where the distance between the grid point and the $m^{\text {th }}$ reference value is designated as the variable $d_{m}$, and the length scale, $c_{x}$ and $c_{y}$ control the rate of fall-off of the weighting function with the different length scales in each of the $x$ and $y$ directions. The results at each step provide the background fields, which are same to the original location, $D^{1,2,3}\left(x_{m}, y_{m}\right)$ and $\gamma$ is a numerical convergence parameter that controls the difference between the weights at each step in the range of 0 to 1 . It should be noted that the eq. (3) becomes the objective function, and the elliptical formula in the square brackets of eq. (4) becomes the constrained function of the graphical optimization.
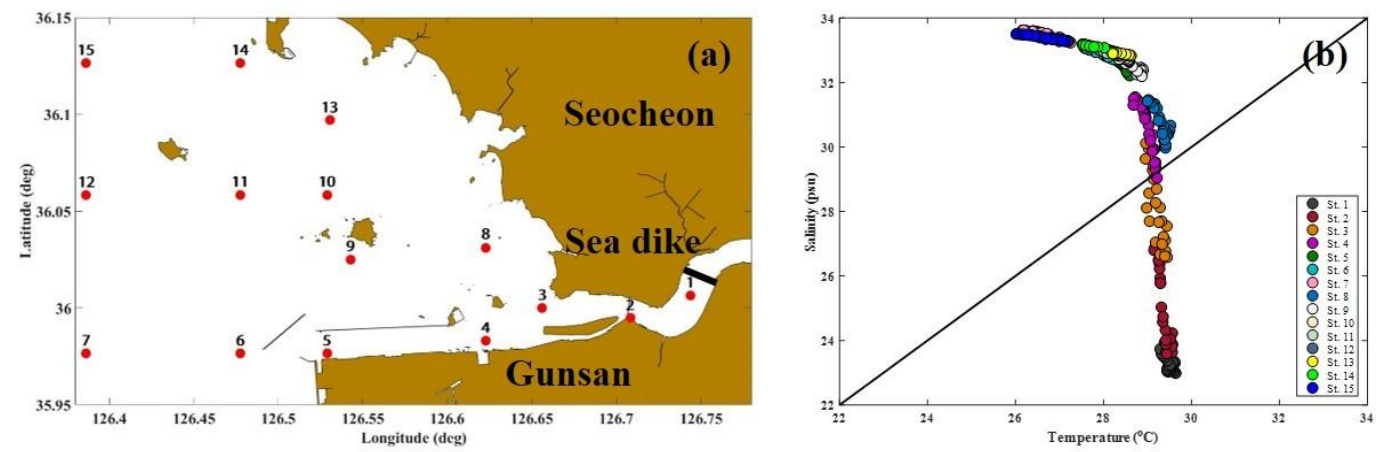

Figure 2: (a) Location of data extracted from the numerical model and (b) correlation coefficient between salinity and temperature 


\section{Results and discussion}

For testing the present scheme of the optimization, we chose the correlation coefficient between temperature and salinity extracted from the 24-hours numerical model [Figure 2]. The two variables have a negative correlation of -0.6 near the sea dike (St. 1) and increase (strong negative correlation) toward the ocean side (St. 7, St. 12, St. 15). Salinity and temperature are not well correlated near the gate and show the stronger relationship to the open ocean. It means that the sources or origins of the salinity and temperature are different. In particular, the GRE has large variations and irregularities of the salinity due to the artificial freshwater discharges but the temperature is strongly controlled by the seasonality rather than human controls.

Figure 3(a) shows the spatial distribution constructed by the original objective function (domain) and inequality constraint region (red ellipse) of the correlation coefficient between temperature and salinity of 24-hours numerical model data. The radius of the ellipse is $20.9 \mathrm{~km}$ in the $x$ direction and $10.5 \mathrm{~km}$ in the $y$ direction, respectively. Such an influence area of the weighting function consists of the elliptical radius and the Gaussian distribution. Therefore, an influence radius may be thought of as that radius where $w_{m}=e^{-1}[14]$ and could be solved by applying nonlinear curve-fitting of the LevenbergMarquardt least square sense.

Figure 3(b) and 3(c) show the spatial distributions of the augmented function for the penalty multipliers $r_{g}=1$ and $r_{g}=25$, respectively. As penalty multiplier $\left(r_{g}\right)$ increases [Figure 3(b) and (c)], the penalty function gradually converges to zero, and the value when the difference of augmented function or design value becomes smaller than the tolerance is the optimal solution. It means that the higher penalty multiplier increases the accuracy of the convergence rate [4, 13, 18]. Therefore, the optimal solution can be estimated by combining the objective function within the constrained function using the transformation method. As a result, the optimal solution in the constraint region is at latitude 36.0871 and longitude 126.3736 as design variables and -0.9782 of the correlation coefficient, which is terminated after 25 loops.
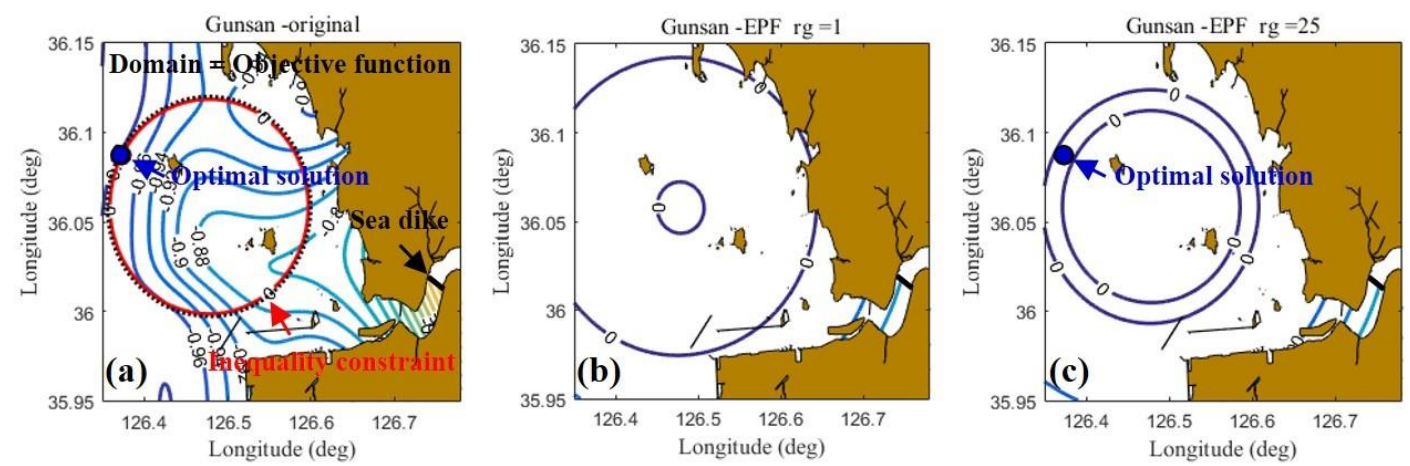

Figure 3: Spatial distribution of (a) the original objective function (domain) and constraint (red ellipse: looks like a circle because of figure size) and (b) the augmented function for the penalty multiplier $r_{g}=1$, and (c) $r_{g}=25$. 


\section{Conclusions}

This study proposes a protocol how to construct the optimal monitoring location considering the temporal and spatial characteristics of an area. Most monitoring systems have been designed in the intuitive sense $[1,3,7]$. For this reason, the short- or long-term monitoring system which is currently operated was not determined in accordance with the reasonable engineering basis, so the reliability of the measurement data may be somewhat lower. Therefore, an appropriate framework for designing the optimized monitoring locations is necessary. In particular, in order to perform optimization in a very complex coastal line such as GRE, it is necessary to develop relatively complex and difficult modules such as constraint optimization technique. However, the present study tried to foc us on developing easier and more robust modules. The development of such an effective optimal monitoring design technique can minimize cost and times for the field observations. Moreover, this may have the additional benefit of improving the accuracy of the hydrodynamic models such as data assimilation $[6$, 17].

In order to get the optimal design of monitoring system, some additional considerations should be considered in the proposed module. First, various kinds of constraints which cover the whole system must be constructed to represent the spatial characteristics of the bay. Secondly, to improve the reliability of the module of present study, the results need to be compared with continuous (real-time) monitoring data. Finally, the index should be developed to include various parameters (e.g. pH, DO, etc.) along with the temperature, salinity, and current through multivariate regression analysis such as an artificial neural network.

\section{Acknowledgement}

This research was a part of the project titled "Development of integrated estuarine management system", funded by the Ministry of Oceans and Fisheries, Korea and the National Research Foundation of Korea (NRF) Grant (No. 2017R1A2B4007977) funded by MSIP of Korea.

\section{References}

[1] Baehr, J., McInerney, D., Keller, K., \& Marotzke, J. (2008). Optimization of an observing system design for North Atlantic meridional overturning circulation. J. Atmos. Oceanic Technol., 25, 625-634.

[2] Barnes, S. L. (1964). A technique for maximizing details in a numerical weather map analysis. $J$. Appl. Meteorol., 3, 396-409.

[3] Barth, N., \& Wunsch, C. (1990). Oceanographic experiment design by simulated annealing. J. Phys. Oceanogr., 20, 1249-1263.

[4] Bhatti, M. A. (2000). Practical optimization methods with mathematica applications. New York: Springer Science+Business Media.

[5] Cho, J., Song, Y., \& Kim, T. I. (2016). Numerical modeling of estuarine circulation in the Geum River Estuary, Korea. Proceedia Eng., 154, 982-989.

[6] Hackert, E. C., Miller, R. N., \& Busalacchi, A. J. (1998). An optimized design for a moored instrument array in the tropical Atlantic Ocean. J. Geophys. Res., 103, 7491-7509.

[7] Hernandez, F., Le Traon, P. Y., \& Barth, N. (1995). Optimizing a drifter cast strategy with a genetic algorithm. J. Atmos. Oceanic Technol., 12, 330-345.

[8] Hwang, J. H., Pham, V. S., Choi, H. J., Chang, J. S., \& Kim, Y. H. (2014). The physical processes in the Yellow Sea. Ocean Coastal Manage., 102, 449-457. 
[9] Jang, D., \& Hwang, J. H. (2013). Estuary classification method for considering climate change effects in South Korea. J. Coastal Res., SI65, 962-967.

[10] Jang, D., Hwang, J. H., Park, Y. G., \& Park, S. H. (2012). A study on salt wedge and river plume in the Seom-Jin River and Estuary. KSCE J. Civ. Eng., 16(4), 676-688.

[11] Kalyanmoy, D. (2012). Optimization for engineering design: Algorithms and example, second ed. New Delhi: PHI Learning Private Limited.

[12] KIm, N. H., Hwang, J. H., \& Ku, H. (20106). Stratification of tidal influenced navigation channel. J. Coastal Res., SI75, 63-67.

[13] Kim, N. H., Pham, V. S., Hwang, J. H., Won, N. I., Ha, H. K., Im, J., \& Kim, Y. (2018). Effects of seas onal variations on sediment-plume streaks from dredging operations. Mar. Pollut. Bull., 129, 26-34.

[14] Koch, E., DesJardin, M., \& Kocin, P. (1983). An interactive Barnes objective map analys s scheme for use with satellite and conventional data. J. Climate Appl. Meteor., 22, 1487-1503.

[15] Le Traon, P. Y., \& Hernandez, F. (1992). Mapping the oceanic mesoscale circulation: Validation of satellite altimetry using surface drifters. J. Atmos. Oceanic Technol., 9, 687-698.

[16] Park, Y. G., Kim, H. Y., Hwang, J. H., Kim, T., Park, S., Nam, J. H., \& Seo, Y. K. (2014). Dynamics of dike effects on tidal circulation around Saemangeum, Korea. Ocean Coastal Manage., 102, 572-582.

[17] Pham, V. S., Hwang, J. H., \& Ku, H. (2016). Optimizing dynamic downscaling in one-way nesting using a regional ocean modeling. Ocean Model., 106, 104-120.

[18] Venkataraman, P. (2009). Applied optimization with MATLAB programming, second ed. New Jersey: John Wiley \& Sons, Inc., Hoboken. 